
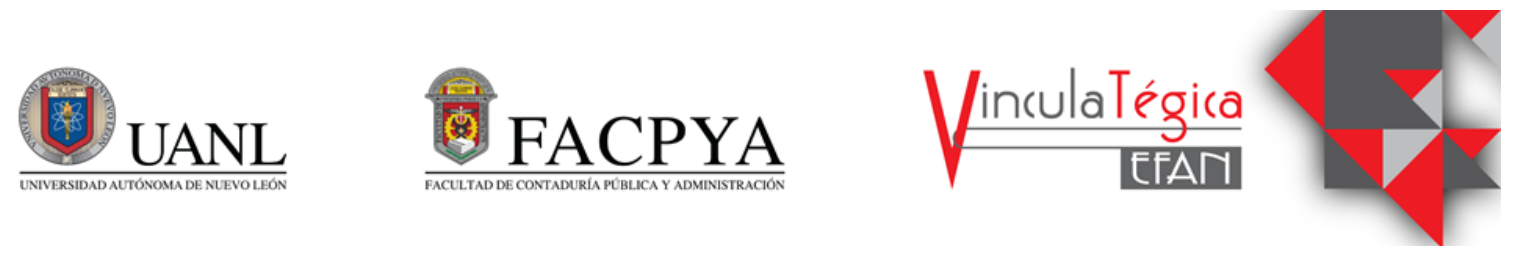

\title{
La adaptación organizacional mediante resiliencia basada en planeación estratégica y cultura de empoderamiento ante el Covid-19
}

\author{
Irving Alberto Varela Bedoya ${ }^{1}$, Joel Mendoza Gómez ${ }^{2}$ y Alexis Jazmín Morán Huertas ${ }^{3}$ \\ 1 Facultad de Contaduría Pública y Administración, Universidad Autónoma de Nuevo León \\ irving.varelabdy@uanl.edu.mx, Niños Héroes, S/N Col. Ciudad Universitaria, San Nicolás de los Garza, N.L, \\ 8183294080. \\ 2 Facultad de Contaduría Pública y Administración, Universidad Autónoma de Nuevo León, \\ joel.mendozagm@uanl.edu.mx, Niños Héroes, S/N Col. Ciudad Universitaria, San Nicolás de los Garza, N.L, \\ 8183294080 . \\ 3 Facultad de Contaduría Pública y Administración, Universidad Autónoma de Nuevo León, \\ alexis.moranhr@uanl.edu.mx, Niños Héroes, S/N Col.Ciudad Universitaria, San Nicolás de los Garza, N.L, \\ 8183294080 .
}

Información del artículo revisado por pares

Fecha de aceptación: junio-2021

Fecha de publicación en línea: diciembre-2021

DOI: https://doi.org/10.29105/vtga7.1-84

\section{RESUMEN}

En la actualidad las organizaciones son afectadas por situaciones que interfieren en su operación habitual; esto genera pérdidas en tiempo y recursos, inclusive podría causar daños al recurso más importante de toda organización, su recurso humano. En ese sentido el Potencial de Resiliencia Organizacional (PRO) entendido como; la capacidad de anticipar amenazas enfrenta efectivamente los eventos adversos al adaptarse a las condiciones cambiantes de tal forma que su implementación beneficiará a las organizaciones que busquen mejorar sus probabilidades para prevalecer ante la adversidad y situaciones de incertidumbre. El objetivo de esta investigación se centra en identificar los precursores del PRO y su influencia mediadora en la Capacidad de Adaptación de las organizaciones a la crisis actual del COVID-19 (CACOV19) bajo un enfoque explicativo. Después de revisar la literatura sobre el tema se aplicó a 126 empresas en el área metropolitana de Monterrey un instrumento de elaboración propia lo que permitió obtener como resultados principales: la Planeación Estratégica (PE) impacta positivamente en el PRO, de tal manera que las organizaciones en la actualidad deben desarrollar su capacidad de anticipación de lo inesperado modelando posibles escenarios para de esta forma planear lo esperado y lo inesperado. Por otra parte, la Cultura de Empoderamiento (CE) impacta positivamente en el PRO; así, las organizaciones necesitan descentralizar correctamente sus recursos humanos y ampliar los poderes de decisión de estos a través de empoderamiento, y de esta forma, aumentar su capacidad de resiliencia.

Palabras clave: Capacidad de Adaptación; COVID19; Cultura de Empoderamiento; Planeación Estratégica; Potencial de Resiliencia Organizacional.

\begin{abstract}
Currently, organizations are affected by situations that interfere with their regular operation; this generates losses in time and resources, it could even cause damage to the essential resource of any organization, its human resources. In that sense, the Organizational Resilience Potential (PRO) understood as; the ability
\end{abstract}


to anticipate threats effectively faces adverse events by adapting to changing conditions in such a way that its implementation will benefit organizations that seek to improve their chances of prevailing in the face of adversity and situations of uncertainty. The objective of this research focuses on identifying the precursors of PRO and its mediating influence on the adaptability of organizations to the current crisis of COVID-19 (CACOV19) under an explanatory approach. After reviewing the literature on the subject, an instrument of their development was applied to 126 companies in the metropolitan area of Monterrey, which allowed the main results to be obtained: Strategic Planning (SP) has a positive impact on the PRO, in such a way that the organizations Nowadays, they must develop their ability to anticipate the unexpected by modeling possible scenarios in order to plan for the expected and the unexpected. On the other hand, the Culture of Empowerment (CE) positively impacts the PRO; thus, organizations need to properly decentralize their human resources and expand their decision-making powers through empowerment, and in this way, increase their resilience capacity.

Keywords: Adaptive Capacity; COVID-19; Culture of Empowerment; Organizational Resilience Potential; Strategic Planning.

JEL Codes: M10, M12, M19.

\section{INTRODUCCIÓN}

El potencial de resiliencia organizacional, entendida como la capacidad para anticipar amenazas, enfrentar efectivamente los eventos adversos y adecuarse a las condiciones cambiantes (Duchek, 2019), permite diferenciar las creencias del pasado en el que se consideraba a las organizaciones robustas con un dominio del mercado tenían los recursos para sobrevivir ante la adversidad. Sin embargo, las teorías modernas que se respaldan en casos de estudio como el de las empresas Kodak Company (Amabile y Kramer, 2012) y Blockbuster LLC, que terminaron en bancarrota, demuestra que no solo se requiere robustez en recursos para sobrevivir. Para esto se requieren las tres características principales de la resiliencia: anticipación, afrontamiento y adaptabilidad (Duchek, 2019). En otras palabras, cuando una organización se prepara para anticipar y mitigar los riesgos que podría enfrentar en un futuro. Se requiere anticipar qué futuro tiene altas probabilidades de suceder, lo que podría nunca llegar a suceder y lo que no se sabe que podría suceder. Existen riesgos que son tan inusuales que pocos administradores los podrían imaginar (Suarez y Montes, 2020). Algunas veces a estos eventos se les llama cisnes negros (black swans), ya que son situaciones que hasta el momento no se tenía conocimiento de su posibilidad de ocurrencia (Kaplan, Leonard \& Mikes, 2020).

Tomando esto en cuenta, el impacto de una crisis (como la generada por la pandemia del COVID-19) repercute en pérdidas de empleo, recorte en salarios, decremento en las ventas, inclusive el cierre de empresas por llegar a la bancarrota. Por otra parte, en estudios previos a este, se ha encontrado que la resiliencia acompaña a las organizaciones en tiempos de crisis (Carvalho y Areal, 2015), permitiéndoles consolidar y prosperar (Duchek, 2019; Somers, 2009), ya que la experiencia de los administradores les permite resolver problemas que han resuelto en el pasado. Aunque, en contraparte, esa misma experiencia podría nublar su visión para prever situaciones improbables e inconcebibles, como el ataque a las torres gemelas de Nueva York, la crisis de las hipotecas subprime en Estados Unidos o el tsunami de Japón. Actualmente, estamos afrontando una nueva situación improbable e inconcebible: la pandemia del síndrome respiratorio agudo (COVID-19) en humanos (Bavel et al., 2020) causada por el nuevo coronavirus SARS-CoV-2, extendida desde Wuhan, China al mundo entero. De allí que, estamos ante una invalorable oportunidad de estudiar la relación que existe entre las organizaciones resilientes y sus capacidades de adaptación a esta crisis del COVID-19 en el contexto mexicano, particularmente las MPYMES de Monterrey y su área metropolitana las cuales también se vieron afectadas por dicha pandemia.

Comprender el impacto de la resiliencia en la CACOV19 les permitiría a las MPYMES beneficiarse de la resiliencia en el nivel organizacional, tal como los académicos han demostrado su utilidad en la psicología, las ciencias naturales, la ingeniería y la ecología antes de extenderse a las ciencias sociales (Carden, Maldonado, y Boyd, 2018) y administrativas. En esta última disciplina se 
pueden conceptualizar herramientas para conocer ¿cómo la PE y la CE son precursores del PRO?, y ¿cómo está a su vez puede mediar el efecto de la CACOV19?

La contribución de este estudio es el diseño de un instrumento, validado en su contenido por expertos y en su fiabilidad, que trata sobre un tema relevante para la gestión de las organizaciones de TIC ya que es de conocimiento general que se encuentran en constante evolución, por tanto, están en un ambiente favorable para desarrollar la resiliencia. Puede ser utilizado, tal cual o modificado por los responsables de la gestión de los procesos en los niveles directivos, gerenciales y mandos medios para determinar si evalúan el desempeño de los procesos a su cargo de forma integral, o identificar a qué elementos deben prestar más atención. Conocimiento que utilizado como herramienta en las organizaciones permite mitigar la vulnerabilidad e incertidumbre ante los eventos adversos para sobrevivir y prosperar ante ellos, siendo el objetivo de la presente investigación.

\section{MARCO TEÓRICO}

La CACOV19 consiste en tener un plan de acción previo a la pandemia, para poner en funcionamiento políticas para trabajar desde casa, tomar medidas de seguridad cibernética y garantizar que los procesos clave puedan continuar operando a distancia (Elliott y Thomas, 2020). Este constructo mide la capacidad de adaptación en la perspectiva de los empleados de una organización ante estas extraordinarias circunstancias y qué tan optimistas se encuentran ante el panorama económico que le espera a la organización para el próximo año.

Los resultados indican que mientras algunas industrias del transporte, la minería, la electricidad y el medio ambiente se han visto afectadas negativamente por la pandemia, otras industrias han sido más resilientes. Algunas de ellas, como son las manufactureras, de educación, atención médica, así como las de Tecnología de la Información y Comunicaciones (TIC), han sido beneficiadas por esta situación (He, Sun, Zhang, y Li, 2020). Así estas organizaciones realizaron procedimientos en la pandemia del COVID-19 para permitir que el personal trabaje desde su hogar con medidas de seguridad cibernética aceptables. También adaptar las actividades laborales para garantizar que los procesos operativos claves pudieran continuar siendo realizados de tal forma de permitir que la organización tuviera una perspectiva optimista desde su situación actual hacia el panorama de incertidumbre del siguiente año.

Las empresas de tecnología se encuentran en un proceso de mejora continua, desde esa perspectiva inicia la capacidad de adaptación de la organización y a su vez la velocidad en la que realiza este cambio (Pettersen y Schulman, 2019). Así es como los modelos teóricos de la resiliencia organizacional frecuentemente mencionan la relación que tiene esta con la adaptación a los cambios e inclusive a los eventos adversos (Ince, Imamoglu, Karakose, y Turkcan, 2017). Cada investigador etiqueta de forma distinta el potencial de resiliencia organizacional, la pueden nombrar como: capacidad de resiliencia, potencial de resiliencia, resiliencia latente $\mathrm{u}$ organización resiliente, por tanto, existen numerosas definiciones independientes, ambiguas y parcialmente inconsistentes del constructo (Duchek, 2019). El uso de la palabra resiliencia tiene sus inicios en el campo de las ciencias naturales. En 1973, el ecologista Holling introduce el término de resiliencia en la ecología y el ecosistema como la capacidad de absorber el cambio y continuar subsistiendo, siendo la habilidad para volver a su equilibrio después de un impacto temporal. Como un complemento a esta definición, Cumming et al. (2005) definieron la resiliencia en la ecología como la capacidad de un sistema para conservar su identidad al hacer frente a un cambio interno a causa de un evento externo.

Otras definiciones las proporcionan; Mallak (1998) quien define la resiliencia latente como la capacidad de una organización para diseñar y aplicar métodos adaptativos positivos que coincidan con la situación inmediata, mientras soporta un estrés mínimo. Por su parte, Somers (2009) define el PRO como el ajuste necesario para garantizar la continuidad operativa de la organización ante una crisis, basado en el desarrollo de la resiliencia latente en las organizaciones; es decir, la resiliencia que actualmente no es evidente o realizada. En la presente investigación se define el PRO como la capacidad para anticipar amenazas, enfrentar 
efectivamente los eventos adversos y adecuarse a las condiciones cambiantes (Duchek, 2019). De esta forma, se conservan las características de la resiliencia latente (Mallak, 1998), conocida como la resiliencia que actualmente no es evidente (Somers, 2009).

Existen investigaciones donde se ha utilizado la resiliencia organizacional para mediar el efecto entre variables independientes con otra variable dependiente, tal es el caso del estudio que se llevó a cabo en hoteles de 3 estrellas, complejos turísticos y casas de huéspedes premium en dos ciudades de Java Oriental, Indonesia utilizado el método del muestreo intencional aplicado a 70 administradores, gerentes generales $y$ gerentes de recursos humanos de estas instituciones. Este resultado reveló que ocurrió una mediación parcial sobre el efecto de la cultura organizacional en el desempeño organizacional. Esta mediación parcial permite inferir que la resiliencia organizacional no era el único mediador en la relación entre cultura organizacional y desempeño organizacional, sino otros mediadores (Suryaningtyas, Sudiro, Troena, e Irawanto, 2019), es así que se observa lo mucho que falta estudiar esta variable y su relación con otras variables tanto independientes como dependientes. Estos conceptos permiten que la resiliencia pueda ayudar a las empresas a sobrevivir y prosperar, al desarrollar un buen plan de acción que permita construir un puente desde las dificultades del presente hacia un futuro de prosperidad, justificando de esta manera su función como variable mediadora. Por otra parte, la PE como concepto surge de la terminología de las ciencias militares y posteriormente fue adoptada por las ciencias sociales (Gavriilidis y Metaxas, 2017). En ese sentido la PE se define en este artículo como la gestión de la vulnerabilidad en los bienes de una organización, a través del desarrollo de posibles escenarios (Lee, Vargo, \& Seville, 2013). En el modelo teórico de Ince et al., (2017) la planeación estratégica forma parte de un mecanismo de ajuste que ayuda a obtener una ventaja competitiva y para dirigir el crecimiento de la organización.

Es importante mencionar la relación entre la PE y la resiliencia para lo cual Somers (2009) planteó la posible existencia de una relación causal entre la planeación de crisis y los comportamientos adaptativos del PRO. Su objetivo principal fue crear estructuras y procesos organizacionales que desarrollan el PRO. Para probar esto, aplicó un cuestionario a 96 directores de obras públicas en los estados de Arizona, Nuevo México, Oklahoma y Texas en Estados Unidos. Con los datos recolectados realizó un análisis de regresión múltiple e identificó que la planeación influye positivamente en la resiliencia $(\beta=.28, p<.01)$. Su modelo empírico es una aportación teórica al PRO, en particular su relación con la planeación.

Por otra parte, los negocios que sobreviven tienen ciertas creencias y un propósito más allá de la acumulación de riquezas (Coutu, 2002), siendo el capital humano uno de sus principales intereses. Es por lo que es importante estudiar la CE. Se define como valores compartidos, creencias o percepciones de los integrantes de la organización para ejercer la autoridad de acceso y control sobre los recursos materiales, humanos y sociales (Freire, 2018; Robbins y Coulter, 2007). Una organización con CE respalda la noción de que todos los empleados se harán cargo de sus entornos de trabajo (Sigler y Pearson, 2000) permitiendo una estructura organizacional descentralizada.

Williams et al. (2016) estudiaron la resiliencia y su relación con el empoderamiento organizacional. Su objetivo fue estudiar la resiliencia entre las enfermeras de cuidados a largo plazo y su relación con el empoderamiento organizacional, aplicando 130 encuestas en América del Norte en los Estados Unidos. Aunque este estudio concluye que el empoderamiento no influye en la resiliencia organizacional, aquí se menciona como un área de oportunidad para profundizar la investigación de esta relación. Así mismo, en otra investigación empírica Abdelaziz Elgamal (2018) se planteó como objetivo estudiar la relación entre la capacidad de resiliencia organizacional y el empoderamiento de los recursos humanos. Los datos se recopilaron mediante un cuestionario de escala tipo Likert de siete puntos. Los sujetos eran profesionales y personal de recursos humanos que trabajaba en 19 organizaciones kuwaitíes. Estas organizaciones operan en los campos de servicios financieros y tecnologías de la información. El número de encuestados fue de 177 sujetos. Identificó que el empoderamiento 
impacta positivamente la resiliencia organizacional $(\beta=.19, \mathrm{p}<.05)$.

Debemos hacer notar que la revisión de la literatura muestra pocos estudios empíricos que relacionen la $\mathrm{CE}$ con el $\mathrm{PRO}$, como consecuencia invita a reflexionar si esta escasez es ocasionada por ser poco significante o una posible brecha teórica. También es interesante profundizar en la relación que existe con la resiliencia y la CACOV19. Ya que, a medida que el mundo científico, en este momento, está acelerando los esfuerzos para luchar contra la pandemia del COVID-19, las empresas de tecnología han sido beneficiadas por este acontecimiento. Tal es el caso, de las impresoras tridimensionales que están facilitando soluciones creativas en términos de equipos de protección personal, equipos médicos como ventiladores, repuestos para dispositivos respiratorios, herramientas de salud $\mathrm{y}$ de bienestar que ayuden a la higiene personal para crear un entorno seguro a los seres humanos (Singh, Prakash, y Ramakrishna, 2020).

Por lo tanto, con base en este marco teórico, se pondrán a prueba las siguientes hipótesis:

- Hipótesis 1. La Cultura de Empoderamiento impacta positivamente en el Potencial de Resiliencia Organizacional.

- Hipótesis 2. La Planeación Estratégica influye positivamente en el Potencial de Resiliencia Organizacional.

- Hipótesis 3. El Potencial de Resiliencia Organizacional incide positivamente en la capacidad de adaptación de las organizaciones a la crisis actual del COVID-19.

\section{MÉTODO}

A continuación, se describe la forma en la cual se llevó a cabo la presente investigación, sus propiedades, características, métodos utilizados, el tipo y diseño de investigación.

Se utilizó el método cuantitativo como lo hizo Godwin y Umoh (2013) para verificar si existe una relación positiva y significativa entre las variables independientes propuestas anteriormente con el PRO y este a su vez con la CACOV19.

Finalmente, se buscó probar el modelo explicativo de las relaciones entre las variables independientes con el PRO como lo propusieron Hayes, Douglas, y Bonner (2014). Con este modelo se determinó las posibles causas y consecuencias del potencial de resiliencia para generar un sentido de entendimiento de este fenómeno.

El diseño fue no experimental por su dimensión temporal, siendo esta transeccional al evaluar el fenómeno y su relación entre un conjunto de variables en un momento dado recolectando datos en un tiempo único (Hayes et al., 2014), en este caso de mayo a junio de 2020. En la presente investigación se describe la relación entre las distintas variables en un momento único determinado en términos correlacionales y en función de la relación causaefecto. Las técnicas por medio de los cuales se desarrolló la investigación fueron: documental, bibliográfica y de campo (Sáenz y Rodríguez, 2014).

Para generar conocimiento a través de la investigación es muy importante medir y cuantificar. Es necesario asignar números a la unidad de análisis acorde a diferentes niveles de calidad en representación del concepto a medir (DeVellis, 2016). Por esta razón, se procedió a operacionalizar las variables, realizar la validez de contenido y elaborar el instrumento de medición (encuesta).

Se llevó a cabo un proceso de abstracción para incorporar diversos elementos mediante indicadores definidos en el proceso de medición (Mendoza y Garza, 2009). A través de la operacionalización de las definiciones mencionadas en la revisión de la literatura y con los ítems obtenidos de los instrumentos de Campos, (2015), Lee et al., (2013), Mallak, (1998b), y Şengül et al., (2019).

Para la validez de contenido, se procedió a solicitar la colaboración de 5 expertos en el área de dirección y administración de empresas. La prueba de validez se realizó de acuerdo con el método de Mendoza y Garza (2009) para evaluar el grado de relevancia de cada ítem. Los resultados de la operacionalización y validación de los expertos fueron 22 afirmaciones (ítems) de intervalo: 8 para medir PRO, 6 de PE, 4 de CE y 5 para la CACOV19. También se aplicaron 7 ítems nominales.

El instrumento elaborado tuvo como objetivo medir la relación esencial entre los 
indicadores y la respuesta observable de los constructos no observables para fundamentar una explicación. El desarrollo del mismo permite el análisis de los indicadores y así generar como resultado algunas inferencias entre los conceptos no observables del estudio (Carmines y Zeller, 1979).

El objeto de estudio son las organizaciones de TIC ya que es de conocimiento general que se encuentran en constante evolución, por tanto, están en un ambiente favorable para desarrollar la resiliencia. Con base al Marco Geoestadístico del INEGI (2000) la zona metropolitana de Monterrey está constituida por los municipios de Apodaca, García, San Pedro Garza García, General Escobedo, Guadalupe, Juárez, Monterrey, Salinas Victoria, San Nicolás de los Garza y Santa Catarina. En esta área metropolitana a través del Directorio Estadístico Nacional de Unidades Económicas (DENUE) se identificaron, y ubicaron, los negocios TIC actualizados al mes de mayo de 2020. El tamaño de la población es de 393 Micro, Pequeñas y Medianas Empresas (MPYMES) con un tamaño de la muestra 126 empresas, dicho cálculo fue realizado a través de la aplicación de Excel utilizando la fórmula para calcular el tamaño de muestra " $n$ " de una variable, desarrollada por Rositas (2014).

Los informantes son los líderes de las organizaciones, a los que se les aplicó el instrumento, ya que son ellos los responsables de desarrollar dentro de su organización los factores que causan del PRO. De esta forma, se recabaron 126 encuestas para su análisis mediante formularios electrónicos enviados por correo electrónico y la red social LinkedIn. Las características demográficas de estos informantes son: el $80 \%$ de ellos hombres y el $20 \%$ mujeres. El $8 \%$ estudiaron hasta preparatoria, el $67 \%$ licenciatura, $29 \%$ maestría y solo una persona un doctorado $(0.8 \%)$. Estos líderes pertenecen a empresas del sector de TIC, donde el $39 \%$ de las organizaciones que participaron tienen más de 20 años de antigüedad en el mercado, mostrando una madurez importante de las organizaciones. El 27 $\%$ de las empresas encuestadas tienen menos de 30 empleados y se identificó que el $49 \%$ de las empresas facturaron menos de 50 millones de pesos en el ejercicio fiscal 2019.

Se realizó un análisis de ecuaciones estructurales ajustadas por mínimos cuadrados parciales (PLS-SEM por sus siglas en inglés) como método estadístico para especificar, estimar e interpretar el modelo explicativo de la variable dependiente en función de la variable mediadora con las variables independientes (Henseler et al., 2014). Para que el análisis funcionara de una manera efectiva se trabajó con variables independientes entre ellas, para de esta forma así determinar si la variable dependiente está siendo influenciada por la variable mediadora y las variables independientes. Esto permitió obtener un valor significativo en las relaciones de tal manera que las hipótesis fueran apoyadas empíricamente e interpretarse como un factor que ayudó a obtener la mayor explicación posible de las variables independientes (Treviño-Saldivar, 2014).

Para determinar la confiabilidad del instrumento se analizó la fiabilidad de consistencia interna (TABLA 1) y se pueden observar los resultados del análisis. Las Alfa de Cronbach, los coeficientes de confiabilidad rho A y la fiabilidad compuesta, estando dentro del rango (0.70-0.90) de lo recomendable, También se calculó el promedio de la varianza extraída (AVE por sus siglas en inglés) donde se obtuvieron resultados superiores a 0.5 , lo que indica que el constructo explica más de la mitad de la varianza de los indicadores (Hair, Risher, Sarstedt, \& Ringle, 2019). Esto infiere que los resultados obtenidos son fiables.

TABLA 1. Fiabilidad de Consistencia Interna

\begin{tabular}{|l|c|c|c|c|}
\hline & $\begin{array}{c}\text { Alfa de Cronbach } \\
0.7-0.9\end{array}$ & $\begin{array}{c}\text { rho_A } \\
0.7-0.9\end{array}$ & $\begin{array}{c}\text { Fiabilidad } \\
\text { compuesta } \\
0.7-0.9\end{array}$ & $\begin{array}{c}\text { AVE } \\
>0.5\end{array}$ \\
\hline CACOV19 & 0.772 & 0.833 & 0.848 & 0.541 \\
\hline CE & 0.719 & 0.739 & 0.826 & 0.545 \\
\hline PE & 0.909 & 0.913 & 0.932 & 0.734 \\
\hline
\end{tabular}




\begin{tabular}{|c|c|c|c|c|}
\hline PRO & 0.866 & 0.874 & 0.896 & 0.521 \\
\hline \multicolumn{3}{|c|}{ Fuente: Elaboración propia con resultados del análisis de fiabilidad mediante el algoritmo de mínimos cuadrados parciales. } \\
\cline { 2 - 2 }
\end{tabular}

\section{RESULTADOS}

La estadística inferencial consiste en obtener conclusiones de un conjunto de datos, usando las herramientas de la Teoría de la Probabilidad. El análisis multivariante es un conjunto de métodos estadísticos y matemáticos que tienen como objetivo analizar resultados para describir e interpretar las observaciones multidimensionales que provienen de más de una variable (MartínCastejón, Lafuente-Lechuga, y Faura-Martínez, 2015). Los resultados obtenidos a través del proceso de investigación, mediante un esmerado manejo de información que fue captada a través la aplicación del instrumento de medición, procesados mediante el análisis en el programa SmartPLS 3.0. Posteriormente, se analizaron los datos para la comprobación de hipótesis.

Asimismo, se realizó un análisis de validez discriminante que permite realmente distinguir un constructo de otro de forma empírica, esto permite establecer que cada constructo es único y de esta forma explicar el fenómeno sin ser confundido con los otros constructos que forman parte del modelo. A través del heterotrait-monotrait ratio (HTMT), es posible evaluar la validez discriminante que se define como el valor medio de las correlaciones de los elementos entre los constructos en relación con la media (geométrica) de las correlaciones promedio para los elementos, de esta forma, determinar si cada constructo es único (HTMT < $0.85)$, si son constructos similares $(0.85<$ HTMT $<0.90$ ), o si son el mismo constructo (HTMT = 1). En la TABLA 2 se observa que no hay resultados de HTMT $=1$, esto quiere decir que ninguno de estos constructos es estadísticamente el mismo constructo, también se observa en la relación que mide la CACOV19 con la variable mediadora de PRO se obtuvo un HTMT $=0.804$ por lo que permite inferir que son constructos distintos (Hair et al., 2019).

TABLA 2. Validez Discriminante (HTMT)

\begin{tabular}{|l|l|l|l|}
\hline & $\begin{array}{l}\text { CACOV19 } \\
<0.9\end{array}$ & $\begin{array}{l}\text { CE } \\
<0.9\end{array}$ & $\begin{array}{l}\text { PE } \\
<0.9\end{array}$ \\
\hline CE & 0.781 & & \\
\hline PE & 0.827 & 0.833 & \\
\hline PRO & 0.824 & 0.869 & 0.875 \\
\hline
\end{tabular}

Fuente: Elaboración propia con resultados del análisis de validez discriminante mediante el algoritmo de mínimos cuadrados parciales.

A continuación, se analizó el supuesto de

la no colinealidad, el cual implica que las variables precursoras no estén correlacionadas entre sí, para esto se utiliza el Factor de Inflación de Varianzas (FIV), donde el nivel de tolerancia

indica que el resultado obtenido de este análisis debe ser menor a 3. En la TABLA 3 se puede observar que todos los valores están dentro del intervalo de confianza.

TABLA 3. Colinealidad del Modelo Estructural (FIV)

\begin{tabular}{|l|l|l|}
\hline & $\begin{array}{l}\text { CACOV19 } \\
<3\end{array}$ & $\begin{array}{l}\text { PRO } \\
<3\end{array}$ \\
\hline CE & & 1.867 \\
\hline PE & & 1.867 \\
\hline PRO & 1.000 & \\
\hline
\end{tabular}

Fuente: Elaboración propia con resultados del análisis de los valores FIV del modelo estructural con el algoritmo de mínimos cuadrados parciales.

El tamaño del efecto del modelo $\left(f^{2}\right)$ mide el impacto relativo de una variable predictora en la variable de respuesta. Se consideran efectos pequeños los resultados de $\mathrm{f}^{2}$ mayores a 0.02 , efectos moderados cuando son superiores a 0.15 y efectos grandes al exceder 0.35 . Los resultados obtenidos con el método de PLS-SEM permiten inferir que la $\mathrm{CE}\left(\mathrm{f}^{2}=.138\right)$ tiene un efecto 
moderado sobre el PRO y la PE $\left(\mathrm{f}^{2}=.555\right)$ tiene un efecto grande sobre el PRO, que a su vez tiene un efecto grande $\left(\mathrm{f}^{2}=.920\right)$ en el CACOV19 (véase figura 1). Otro indicador importante es la varianza explicada $\left(\mathrm{R}^{2}\right)$ de la variable de respuesta del modelo estructural, esto ya que a mayor valor de $\mathrm{R}^{2}$, quiere decir que mayor será capacidad explicativa de las variables predictoras a las variables de respuesta. Los resultados revelan que la CE y la PE explican el $66.7 \%$ $\left(\mathrm{R}^{2}=.667\right)$ de la varianza del PRO, que a su vez explica el $47.9 \%\left(\mathrm{R}^{2}=.479\right)$ de la varianza de la CACOV19 (véase FIGURA 1).

Adicionalmente se requiere determinar el criterio de relevancia predictiva $\left(\mathrm{Q}^{2}\right)$ de StoneGeisser que se obtiene mediante el procedimiento de blindfolding considerando un umbral superior de 0.15 para una relevancia predictiva moderada y un umbral superior de 0.35 para una relevancia predictiva fuerte (Hair, Risher, Sarstedt, y Ringle, 2019). En este caso la CE y la PE presentan una relevancia predictiva moderada hacia el PRO de $33.6 \%\left(\mathrm{Q}^{2}=.336\right)$ que a su vez muestra una relevancia predictiva moderada hacia la CACOV19 de $24.9 \%\left(\mathrm{Q}^{2}=.249\right)$. Referente a la magnitud del impacto se deduce que la $\mathrm{CE}$ $(\beta=.293, p<.001)$ y la $\operatorname{PE~}(\beta=.588, p<.001)$ influyen positivamente en el PRO, que a su vez el efecto mediador del PRO $(\beta=.692, \mathrm{p}<.001)$ impacta positivamente la CACOV19 (véase FIGURA 1).

FIGURA 1. Modelo Gráfico de Hipótesis Finales Aceptadas

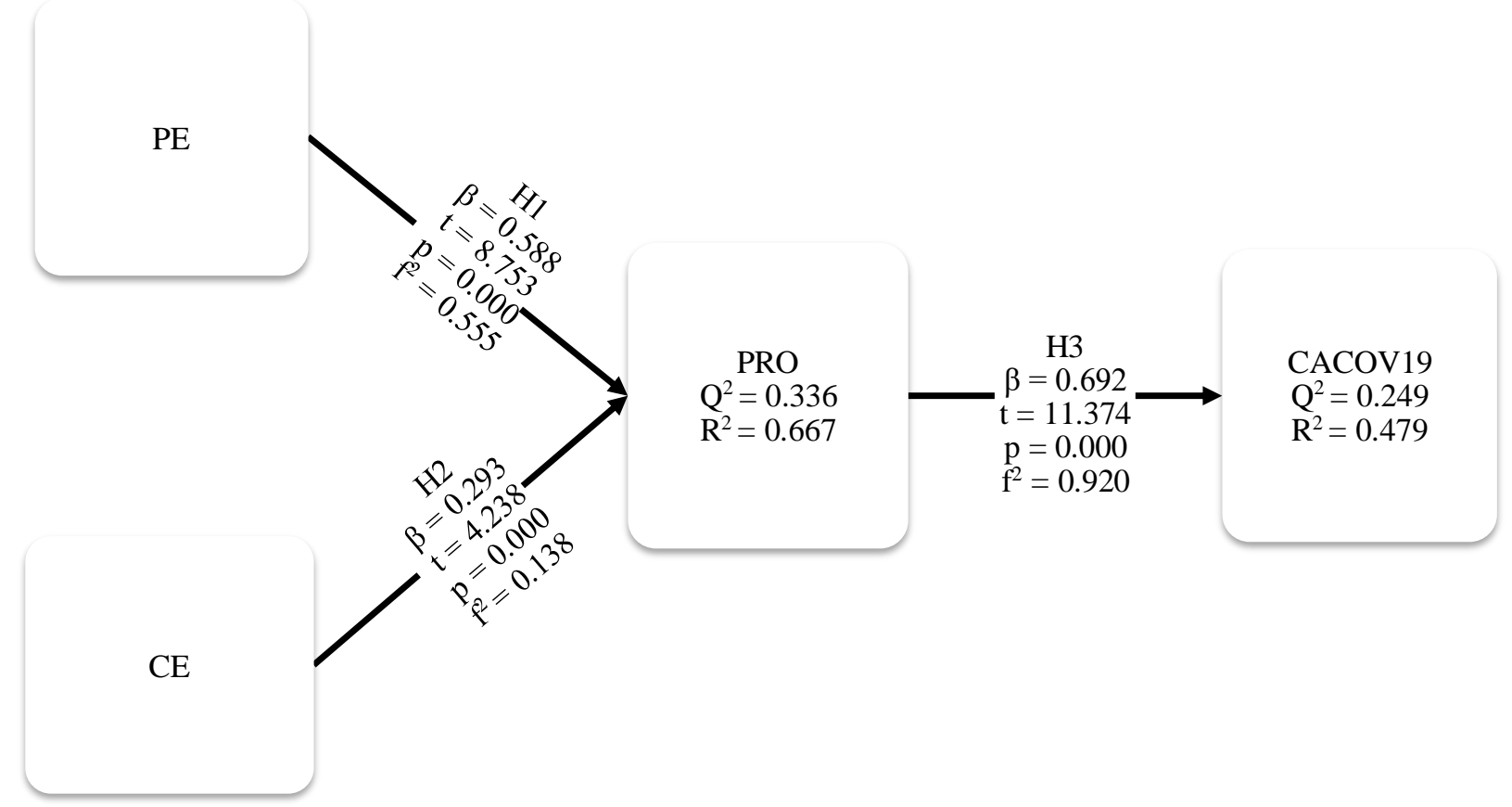

Fuente: Elaboración propia del autor con resultados del análisis del modelo estructural con el algoritmo de mínimos cuadrados parciales. (PE = Planeación Estratégica; CE = Cultura de Empoderamiento; PRO = Potencial de Resiliencia Organizacional; CACOV19= Capacidad de Adaptación al COVID-19).

Se puede observar la comprobación de las hipótesis siendo estadísticamente significativas, así como la variable de PRO realiza un efecto mediador entre la $\mathrm{CE}(\beta=.203$, $\mathrm{P}<.001)$ y la $\mathrm{PE}(\beta=.407, \mathrm{P}<.001)$ con la CACOV19 (ver TABLA 4). 
TABLA 4. Comprobación de Hipótesis (efectos totales)

\begin{tabular}{|c|c|c|c|c|}
\hline \multirow{6}{*}{$\begin{array}{l}\mathrm{H} 1 \\
\mathrm{H} 2 \\
\mathrm{H} 3\end{array}$} & VARIABLES & $\beta$ & $\mathrm{t}$ & Sig. \\
\hline & CE-> PRO & 0.293 & 4.238 & 0.000 \\
\hline & PE -> PRO & 0.588 & 8.753 & 0.000 \\
\hline & $\begin{array}{r}\text { PRO -> CACOV19 } \\
\text { Varia }\end{array}$ & $\begin{array}{l}0.692 \\
\text { or (hipc }\end{array}$ & $\begin{array}{l}11.374 \\
\text { is implíci }\end{array}$ & 0.000 \\
\hline & CE $->$ CACOV19 & 0.203 & 3.904 & 0.000 \\
\hline & PE -> CACOV19 & 0.407 & 6.311 & 0.000 \\
\hline
\end{tabular}

Fuente: Elaboración propia con resultados del análisis bootstrapping con el algoritmo de mínimos cuadrados parciales.

Dado que todas las relaciones entre constructos tienen magnitudes significativas ( $p$ $<0.001)$ permite que los resultados apoyen las tres hipótesis. En primer lugar, H1 establece que existe un impacto positivo y significativo entre la cultura de empoderamiento con el potencial de resiliencia organizacional, por tanto, a mayor $\mathrm{CE}$ se obtiene una mayor explicación del PRO. En segundo lugar, H2 plantea que la planeación estratégica influye positivamente en el PRO, así es como, a mayor PE permite incrementar el PRO. En tercer lugar, H3 apuntala que el potencial de resiliencia organizacional incide positivamente en CACOV19.

\section{CONCLUSIONES}

Este artículo logró el propósito de la investigación y se alcanzaron los objetivos como se mencionaron en el apartado anterior. Aquí se presentan varias contribuciones teóricas significativas al cuerpo de conocimiento existente en la literatura sobre la CACOV19 y constituye una importante contribución práctica. El artículo utiliza modelos teóricos y empíricos existentes como el de Mallak (1998), Lee et al. (2013), Ince et al., (2017), Somers (2009) y Duchek (2019). Además, este artículo aporta valiosos conocimientos empíricos de la relación que existe entre la cultura de empoderamiento y la planeación estratégica para explicar el comportamiento del potencial de resiliencia organizacional que a su vez ayuda a explicar la
CACOV19. También, el aumento de la incertidumbre y los desafíos de la presente crisis global que requiere que los administradores tengan una mejor comprensión teórica del PRO para gestionar eficazmente sus negocios en el turbulento entorno empresarial en el que operan. Para comprender los efectos que influyen en la continuidad de las operaciones y mitigar los efectos negativos provocados por eventos adversos, este artículo proporciona evidencia empírica de los efectos beneficiosos del PRO para adaptarse a cambios disruptivos.

Una vez terminada la investigación las hipótesis acerca de si la Cultura de Empoderamiento impacta positivamente en el Potencial de Resiliencia Organizacional, si la Planeación Estratégica influye positivamente en el Potencial de Resiliencia Organizacional y si el Potencial de Resiliencia Organizacional incide positivamente en CACOV19, fueron corroboradas y constatados por lo cual se concluye que el estudio justificó su desarrollo. Por estas razones, los resultados de la investigación se pueden aplicar a las empresas en sus contextos organizativos específicos ajustando y manipulando las variables según sea necesario para un caso particular.

Se recomienda que la investigación futura debe incorporar otros constructos no incluidos en este estudio para obtener una mejor comprensión a la CACOV19. Este artículo proporciona importantes aportaciones empíricas 
para la teoría de la resiliencia y la adaptación, por tanto, contribuye a la literatura con el desarrollo de un enfoque resiliente y adaptativo en las organizaciones. 


\section{REFERENCIAS}

Abdelaziz Elgamal, M. (2018). Dynamic Organizational Capabilities: The Joint Effect of Agility, Resilience and Empowerment. Journal of Human Resource Management, 6(2), 44. doi: 10.11648/j.jhrm.20180602.11

Amabile, T., \& Kramer, S. (2012). The Human Cost of Kodak's Bankruptcy. Harvard Buiness Review. Recuperado de https://hbr.org/2012/01/the-human-cost-of-kodaks-bankr

Bavel, J. J. Van, Baicker, K., Boggio, P. S., Capraro, V., Cichocka, A., Cikara, M., ... Willer, R. (2020). Using social and behavioural science to support COVID-19 pandemic response. Nature Human Behaviour, 4(5), 460-471. doi: 10.1038/s41562-020-0884-z

Campos, K. (2015). Dimensions of business resilience in the context of post-disaster recovery in Davao City, Philippines. Society of Interdisciplinary Business Research Conference (2015), Review Integrated Business Research (RIBER).

Carden, L. L., Maldonado, T., \& Boyd, R. O. (2018). Organizational resilience: A look at McDonald's in the fast food industry. Organizational Dynamics, 47(1), 25-31. doi: 10.1016/j.orgdyn.2017.07.002

Carmines, E., \& Zeller, R. (1979). Reliability and Validity Assessment. doi: 10.4135/9781412985642

Carvalho, A., \& Areal, N. (2015). Great places to work®: Resilience in times of crisis. 46(1), 71-94. doi: $10.1002 / \mathrm{hrm}$

Coutu, D. (2002). How resilience works. Harvard business review, 80(5), 46-56. doi: 10.1016/j.leaqua.2005.03.003

Cumming, G. S., Barnes, G., Perz, S., Schmink, M., Sieving, K. E., Southworth, J., ... Van Holt, T. (2005). An Exploratory Framework for the Empirical Measurement of Resilience. Ecosystems, 8(8), 975-987. doi: 10.1007/s10021-005-0129-z

DeVellis, R. F. (2016). Scale development: Theory and applications (Vol. 26). Sage publications.

Duchek, S. (2019). Organizational resilience: A capability-based conceptualization. Business Research, 132. doi: 10.1007/s40685-019-0085-7

Elliott, R., \& Thomas, C. (2020). Coronavirus Organizational Preparedness Report 3rd Edition. Bci, 3(May), 1-16. Recuperado de https://www.thebci.org/resource/bci-coronavirus-organizationalpreparedness-report-third-edition.html

Freire, P. (2018). Pedagogy of the oppressed. Bloomsbury publishing USA.

Gavriilidis, G., \& Metaxas, T. (2017). Strategic planning and city/regional development: Review, analysis, critique and applications for Greece. (81131). Recuperado de https://mpra.ub.unimuenchen.de/81131/1/MPRA_paper_81131.pdf

Godwin, I., \& Umoh, E. (2013). Knowledge management and organizational resilience in Nigerian manufacturing organizations. Developing Country Studies, 3(9), 104-120.

Hair, J. F., Risher, J. J., Sarstedt, M., \& Ringle, C. M. (2019). When to use and how to report the results of PLS-SEM. European Business Review, 31(1), 2-24. doi: 10.1108/EBR-11-2018-0203

Hayes, B., Douglas, C., \& Bonner, A. (2014). Predicting emotional exhaustion among haemodialysis nurses: A structural equation model using Kanter's structural empowerment theory. Journal of Advanced Nursing, 70(12), 2897-2909. doi: 10.1111/jan.12452

He, P., Sun, Y., Zhang, Y., \& Li, T. (2020). COVID-19's Impact on Stock Prices Across Different Sectors-An Event Study Based on the Chinese Stock Market. Emerging Markets Finance and Trade, 56(10), 2198-2212. doi: 10.1080/1540496X.2020.1785865

Henseler, J., Dijkstra, T. K., Sarstedt, M., Ringle, C. M., Diamantopoulos, A., Straub, D. W., ... Calantone, R. J. (2014). Common Beliefs and Reality About PLS. Organizational Research Methods, 17(2), 182209. doi: $10.1177 / 1094428114526928$

Holling, C. S. (1973). Resilience and Stability of Ecological Systems. Annual Review of Ecology and Systematics, 4(1), 1-23. doi: 10.1146/annurev.es.04.110173.000245

Ince, H., Imamoglu, S., Karakose, M., \& Turkcan, H. (2017, diciembre 20). The Search For Understanding Organizational Resilience. 230-243. doi: 10.15405/epsbs.2017.12.02.20

Kaplan, R., Leonard, H., \& Mikes, A. (2020). The Risks You Can't Foresee. Harvard Buiness Review, 117. Recuperado de https://hbr.org/2020/11/the-risks-you-cant-foresee 
Lee, A. V., Vargo, J., \& Seville, E. (2013). Developing a Tool to Measure and Compare Organizations' Resilience. Natural Hazards Review, 14(1), 29-41. doi: 10.1061/(ASCE)NH.1527-6996.0000075

Mallak, L. (1998). Measuring resilience in health care provider organizations. Health Manpower Management, 24(4), 148-152. doi: 10.1108/09552069810215755

Martín-Castejón, P. J., Lafuente-Lechuga, M., \& Faura-Martínez, Ú. (2015). Guía práctica de Estadística aplicada a la empresa y al marketing. Ediciones Paraninfo, SA.

Mendoza, J., \& Garza, J. (2009). La medición en el proceso de investigación científica: Evaluación de validez de contenido y confiabilidad. Innovaciones de negocios, 6(11), 17-32.

Pettersen, K. A., \& Schulman, P. R. (2019). Drift, adaptation, resilience and reliability: Toward an empirical clarification. Safety Science, 117, 460-468. doi: 10.1016/j.ssci.2016.03.004

Robbins, S. P., \& Coulter, M. (2007). Principles of management. Translated by Seyyed Mohammad Arabi and Mohammed Ali Hamid Rafiee and Behrouz Asrari Ershad, Fourth Edition, Tehran: Office of Cultural Studies.

Rositas, J. (2014). Los tamanos de las muestras en encuestas de las ciencias sociales y su repercusion en la generacion del conocimiento (Sample sizes for social science surveys and impact on knowledge generation). Innovaciones de Negocios, 11(2), 235-268. Recuperado de http://www.web.facpya.uanl.mx/rev_in/Revistas/11_22/11.22 Art4 pp 235 - 268.pdf

Sáenz, K., \& Rodríguez, K. (2014). Habilidades Investigativas, Capítulo 4. En Métodos y técnicas cualitativas y cuantitativas aplicables a la investigación en ciencias sociales. Tirant Humanidades México.

Şengül, H., Marşan, D., \& Gün, T. (2019). Survey assessment of organizational resiliency potential of a group of Seveso organizations in Turkey. Proceedings of the Institution of Mechanical Engineers, Part O: Journal of Risk and Reliability, 233(3), 470-486. doi: 10.1177/1748006X18802655

Sigler, T. H., \& Pearson, C. M. (2000). Creating an empowering culture: examining the relationship between organizational culture and perceptions of empowerment. Journal of Quality Management, 5(1), 27-52. doi: 10.1016/S1084-8568(00)00011-0

Singh, S., Prakash, C., \& Ramakrishna, S. (2020). Three-dimensional printing in the fight against novel virus COVID-19: Technology helping society during an infectious disease pandemic. Technology in Society, 62, 101305. doi: 10.1016/j.techsoc.2020.101305

Somers, S. (2009). Measuring Resilience Potential: An Adaptive Strategy for Organizational Crisis Planning. Journal of Contingencies and Crisis Management, 17(1), 12-23. doi: 10.1111/j.14685973.2009.00558.x

Suarez, F., \& Montes, J. (2020). Building Organizational Resilience. Harvard Buiness Review, 9-14. Recuperado de https://hbr.org/2020/11/building-organizational-resilience

Suryaningtyas, D., Sudiro, A., Troena, E., \& Irawanto, D. (2019). Organizational Resilience: As Mediating Effect of Organizational Culture and Organizational Performance. Proceedings of the Proceedings of the 1st Sampoerna University-AFBE International Conference, SU-AFBE 2018, 6-7 December 2018, Jakarta Indonesia. doi: 10.4108/eai.6-12-2018.2286329

Treviño-Saldivar, E. (2014). Regresión múltiple, Capítulo 14. En Métodos y técnicas cualitativas y cuantitativas aplicables a la investigación en ciencias sociales. Tirant Humanidades México.

Williams, J., Hadjistavropoulos, T., Ghandehari, O. O., Malloy, D. C., Hunter, P. V., \& Martin, R. R. (2016). Resilience and organisational empowerment among long-term care nurses: effects on patient care and absenteeism. Journal of Nursing Management, 24(3), 300-308. doi: 10.1111/jonm.12311 